\title{
Determinação da Força de Adesão da Metalização com Alumínio em Filmes Plásticos Utilizados em Embalagens Flexíveis - Desenvolvimento e Validação de Metodologia
}

\author{
Léa M. Oliveira, Claire I. G. L. Sarantópoulos, Fábio G. Teixeira \\ Centro de Tecnologia de Embalagem, ITAL \\ Patrícia A. Suguiuti \\ Faculdade de Engenharia de Alimentos, UNICAMP
}

\begin{abstract}
Resumo: Filmes de BOPP e de PET metalizados são muito utilizados em embalagens multicamadas, pois reúnem boas propriedades mecânicas e de barreira a gases e umidade, as quais, bem como a aparência e a integridade, são comprometidas quando ocorre a delaminação da estrutura multicamada. Um dos principais fatores que influem na resistência à delaminação é a força de adesão da camada de alumínio ao substrato polimérico, parâmetro para o qual não há um método de quantificação normalizado. O objetivo deste trabalho foi estabelecer e validar um método de ensaio para determinar esta força de adesão. $\mathrm{O}$ método foi definido usando um planejamento experimental com fatores fixos e variáveis sendo que, para cada variável, foram definidos dois níveis. O método de ensaio estabelecido mostrou-se estável, preciso, robusto e replicável. Consiste, basicamente, na termosselagem de um filme plástico à face metalizada da amostra, em condições específicas de temperatura/tempo/pressão, e medida da força para destacamento da metalização, em ângulo e velocidade específicos, em máquina universal de ensaios.
\end{abstract}

Palavras-chave: Força de adesão, filme plástico metalizado, filme de PET, filme de BOPP.

\section{Adhesion Strength of Aluminum on Metallized Plastic Films Used in Flexible Packages - Development and Validation of a Test Procedure}

Abstract: OPP and OPET metallized films are widely used as substrates in multilayer packages, since they bring together good mechanical properties and barrier to gases and water vapor, which are damaged with delamination of the film layers, together with the visual appearance and package integrity. One of the main factors that affect the package delamination is the adhesion strength of aluminum layer to the polymeric substrate, a parameter for which there is no standard procedure to quantify. The aim of this work was to establish and validate a procedure to determine the adhesion strength of the aluminum to the polymer. This test procedure was carried out using experimental design with fixed and variables parameters with two levels for each variable. The test procedure was stable, robust and replicable. It consisted basically of the heat sealing of a plastic film to the metallized sample surface, under specific temperature/time/pressure conditions and the measuring of adhesion strength of metallization in a universal test machine running at specific angle and speed.

Keywords: Adhesion strength, metallized plastic films, PET film, OPP film.

\section{Introdução}

Filmes de BOPP - polipropileno biorientado e de PET poli(tereftalato de etileno) metalizados apresentam boas propriedades mecânicas e de barreira a gases e a umidade e, portanto, são excelentes opções para conferir propriedades de barreira a materiais laminados com espessura total reduzida. De acordo com Mueller \& Weisser ${ }^{[1]}$, a metalização, que consiste basicamente na deposição de alumínio evaporado sobre o substrato, na forma de uma fina camada (40 a $100 \mathrm{~nm}$ ), aumenta em 20 a 100 vezes as propriedades de barreira do material. Além disto, filmes de BOPP e de PET são considerados substratos ideais para a metalização, devido às suas propriedades superficiais para receber e ancorar a camada de metal e à sua estabilidade dimensional.

Contudo, as propriedades de barreira, a integridade e a aparência deste tipo de embalagem são comprometidas quando ocorre a delaminação (separação das camadas) da estrutura multicamada, o que pode afetar a vida útil e a imagem do produto, além de resultar em perdas econômicas. A resistência à delaminação de um material de embalagem, ou seja, a força necessária para separar as camadas de uma estrutura, é função da capacidade de adesão das duas superfícies. No caso das embalagens flexíveis metalizadas, a resistência à delaminação é definida por fatores como: grau de adesão (ancoragem) do metal ao substrato; tipo e quantidade de tratamentos superficiais aplicados aos substratos; tipo, gramatura e grau de cura do adesivo; migração de aditivos para a superfície do substrato, a exemplo dos deslizantes; presença de impressão, natureza de tintas; homogeneização dos componentes do adesivo; condições de aplicação do adesivo como, por exemplo, a temperatura e a pressão. Alguns componentes do produto acondicionado também são agressivos ao material de embalagem. Os esforços a que a embalagem é submetida durante transporte, distribuição e comercialização do produto também contribuem para a delaminação do filme ${ }^{[2-6]}$.

A força de adesão do metal ao substrato além de afetar a resistência à delaminação da estrutura, também afeta a qualidade da termossoldagem e, consequentemente, o desempenho da embalagem. Estudos têm demonstrado que o aumento da força de adesão da metalização ao substrato também contribui para a melhoria das propriedades de barreira ao oxigênio e ao vapor d'água do filme de PET metalizado. Dependendo do tratamento a que o BOPP é submetido pode-se ter um ganho na força de adesão do metal e melhoria da barreira ao oxigênio da estrutura ${ }^{[7,8]}$.

Autor para correspondência: Léa M. Oliveira, Centro de Tecnologia de Embalagem, Instituto de Tecnologia de Alimento, Av. Brasil, 2880,

CEP 13070-178, Campinas, SP, Brasil. E-mail: leamzov@ital.sp.gov.br 
Diferentes métodos de ensaio foram propostos para a avaliação do grau de adesão da camada de metalização ao substrato polimérico. Basicamente, em todos eles, a face metalizada do filme é termosselada a outro filme polimérico e, posteriormente, determinase a força necessária para separar os materiais e o grau de remoção do alumínio do substrato. As principais diferenças entre os métodos são: o ângulo em que deve ser mantido o corpo-de-prova durante o ensaio, 90 ou $180^{\circ}$; o tipo de filme polimérico termosselado no substrato metalizado e que promove o destacamento do metal; as condições de termosselagem deste filme ao substrato metalizado (tempo, temperatura, pressão, número de mordentes aquecidos na termosseladora); o emprego ou não de fita adesiva para minimizar problemas de alongamento e/ou rasgo de materiais durante o ensaio; e a velocidade em que é determinada a força de adesão no ensaio, que varia em média entre 50 e $300 \mathrm{~mm} / \mathrm{min}^{[7-12]}$.

Trabalhos desenvolvidos no CETEA/ITAL demonstraram que o uso de fita adesiva para aumentar a resistência ao rasgamento do filme metalizado ou minimizar o alongamento do filme termosselante e as características desta fita afetam os resultados da força de adesão. Estes estudos também indicaram que o posicionamento do corpo-de-prova no equipamento (filme metalizado ou filme termosselante na garra móvel), os parâmetros de termosselagem e o tempo decorrente entre a operação de termosselagem do corpo-de-prova e a determinação da força de adesão também podem influenciar os resultados.

As normas que estabelecem os requisitos mínimos de qualidade de filmes de PET e de $\mathrm{BOPP}^{[13,14]}$ não abordam esta questão, contribuindo para a falta de um método de ensaio validado e, consequentemente, a falta de valores de adesão de referência. Sendo assim, o objetivo deste trabalho foi estabelecer e validar um método de ensaio capaz de medir a força de adesão da metalização a filmes de PET e de BOPP, ferramenta fundamental para o estabelecimento de valores de referência para a força de adesão da metalização.

\section{Experimental}

\section{Materiais}

Foram utilizadas 17 amostras de filmes plásticos metalizados produzidos no Brasil. A amostragem compreendeu filmes de BOPP e de PET, homo e copolímeros, sem e com diferentes tratamentos,

Tabela 1. Amostras estudadas.

\begin{tabular}{ccc}
\hline Amostra & Espessura total $(\boldsymbol{\mu m})$ & DO \\
\hline BOPP metalizado 1 & 18 & 2,5 \\
BOPP metalizado 2 & 20 & 2,3 \\
BOPP metalizado 3 & 22 & 1,9 \\
BOPP metalizado 4 & 21 & 2,1 \\
BOPP metalizado 5 & 23 & 2,3 \\
BOPP metalizado 6 & 19 & 2,4 \\
PET coat metalizado 1 & 13 & 2,1 \\
PET coat metalizado 2 & 13 & 2,1 \\
PETcoat metalizado 3 & 13 & 2,1 \\
PET st metalizado 4 & 14 & 2,0 \\
PET st metalizado 5 & 14 & 2,1 \\
PET st metalizado 6 & 13 & 2,1 \\
PET coex metalizado 7 & 13 & 2,1 \\
PET coex metalizado 8 & 13 & 2,1 \\
PET coex metalizado 9 & 13 & 2,1 \\
PET metalizado 10 & 13 & 2,0 \\
PET metalizado 11 & 14 & 2,0 \\
\hline
\end{tabular}

PET coat: filme de PET com tratamento superficial por coating. PET coex: filme de PET com tratamento superficial por coextrusão. PET st: filme de PET sem tratamento. DO: densidade ótica. produzidos em diferentes épocas, por diferentes empresas, de forma que o universo de trabalho fosse representativo do mercado nacional. Na Tabela 1 são apresentadas as amostras utilizadas, sua espessura total e densidade ótica, um parâmetro que indica a quantidade de alumínio depositada no substrato polimérico.

\section{Método de ensaio - planejamento do experimento}

Diversos fatores podem ter influência na determinação da força de adesão da metalização. Com base na literatura e em ensaios preliminares foram selecionados os fatores mais relevantes, que foram divididos em dois grupos, os fixos e os variáveis, sendo que neste último foram concentrados os fatores mais impactantes nos resultados. Para cada um dos fatores variáveis foram definidos dois níveis. As amostras selecionadas, BOPP metalizado 1 e BOPP metalizado 3, foram as que apresentaram a maior e a menor quantidade de alumínio depositado, respectivamente. Na Tabela 2 estão detalhados os fatores e níveis definidos.

A fim de aumentar a precisão do experimento foram utilizadas as técnicas estatísticas de blocagem, fracionamento e hierarquização. A blocagem permitiu a redução do tamanho do experimento e o aumento da sua precisão, sendo um recurso aplicado quando há uma variável que influencia nos resultados, mas que não se deseja estudar. É o caso das amostras, cujo amplo universo permite resultados distintos entre elas e, ainda assim, o método de ensaio deve ser capaz de medir a força de adesão em todas elas. Definiu-se, portanto, dois blocos: BOPP metalizado 1 e BOPP metalizado 3 . Com a blocagem da amostra, restaram ainda cinco variáveis, ou seja, um experimento fatorial $2^{\mathrm{k}}$, onde $\mathrm{k}$ é igual a cinco, o que resulta em interações de $5^{\mathrm{a}}$ ordem que, de acordo com Leão ${ }^{[15]}$, além de serem de difícil interpretação são de pouca utilidade. Por isso foi feito o fracionamento, um recurso muito aplicado quando o número de fatores é superior a quatro, o que reduziu o número de corridas testes e o número de interações àquelas de maior interesse. A hierarquização foi necessária para duas variáveis, o ângulo de ensaio e a temperatura de termosselagem, pois seria inviável a troca constante dos acessórios que fixam o ângulo de ensaio e a alteração da temperatura das barras da termosseladora.

De acordo com Leão ${ }^{[15]}$ os três princípios básicos de um planejamento de experimentos são a blocagem, já comentada, a replicação e a aleatoridade. As réplicas diminuem os erros de medição e o erro experimental, permitindo uma estimativa mais precisa do efeito de um fator nos resultados. A aleatorização das corridas garante uma distribuição uniforme dos fatores fixos, de forma que os erros se tornam variáveis aleatórias, distribuídas independentemente.

Nesta fase do estudo foram realizadas duas réplicas do experimento. Na primeira réplica foram feitas três repetições de cada tratamento e, na segunda, cinco repetições. As medidas da força de adesão foram feitas em uma máquina universal de ensaios Instron, modelo 5500R, operando com célula de carga de $10 \mathrm{~N} \mathrm{e}$ velocidade de $305 \mathrm{~mm} / \mathrm{min}$. Como o objetivo deste trabalho foi definir um método de ensaio que pudesse ser utilizado com diferentes filmes metalizados, foi dada ênfase na variância dos resultados. O delineamento dos experimentos foi feito com o EstatcampExcel_ Plus versão 1.6

\section{Método de ensaio - avaliação da estabilidade/robustez}

A avaliação da estabilidade e da robustez de um método de ensaio faz parte do seu processo de desenvolvimento e validação e indicam sua capacidade de permanecer inalterado, apesar de pequenas variações que possam ocorrer nos parâmetros analíticos/ condições de ensaio como, por exemplo, analista e amostra, entre outros. A robustez de um método é uma indicação de sua confiabilidade durante uso normal ${ }^{[16]}$. 
Tabela 2. Fatores fixos e variáveis do método de ensaio para determinação da força de adesão do metal ao substrato polimérico.

\begin{tabular}{|c|c|c|}
\hline Fatores fixos & Fatores variáveis & Níveis dos fatores variáveis \\
\hline Fita dupla face: $\operatorname{Scotch}^{\circledR}$, código 4274 & Amostra & BOPP metalizado 1 e BOPP metalizado 3 \\
\hline Fita para ancoragem (tipo): BOPP Tartan ${ }^{\circledR}$, código 5899 & Filme selante & EAA e PET/EAA \\
\hline $\begin{array}{l}\text { Termosseladora: Brugger, } 2 \text { barras aquecidas, com perfil plano, } \\
\qquad 20 \mathrm{~mm} \text { de largura, revestidas com teflon }\end{array}$ & Ângulo de delaminação & $90 / 180^{\circ}$ \\
\hline Termosselagem transversal à direção de fabricação do material & Utilização de fita para ancoragem & Sim / Não \\
\hline Condicionamento: $23 \pm 2{ }^{\circ} \mathrm{C} \mathrm{e}(50 \pm 5) \%$ UR por 24 horas & Tempo de termosselagem & $5 / 10$ segundos \\
\hline Ambiente de termosselagem: $23 \pm 2{ }^{\circ} \mathrm{C}$ & - & - \\
\hline Ambiente de ensaio: $23 \pm 2{ }^{\circ} \mathrm{C}$ e $(50 \pm 5) \%$ UR & - & - \\
\hline Velocidade do ensaio: $305 \mathrm{~mm} / \mathrm{min}$ & - & - \\
\hline $\begin{array}{l}\text { Posicionamento do corpo-de-prova na máquina universal de } \\
\text { ensaios: filme metalizado na garra fixa }\end{array}$ & - & - \\
\hline Sequência/método de preparo do corpo-de-prova & - & - \\
\hline
\end{tabular}

EAA: copolímero de etileno e ácido acrílico.
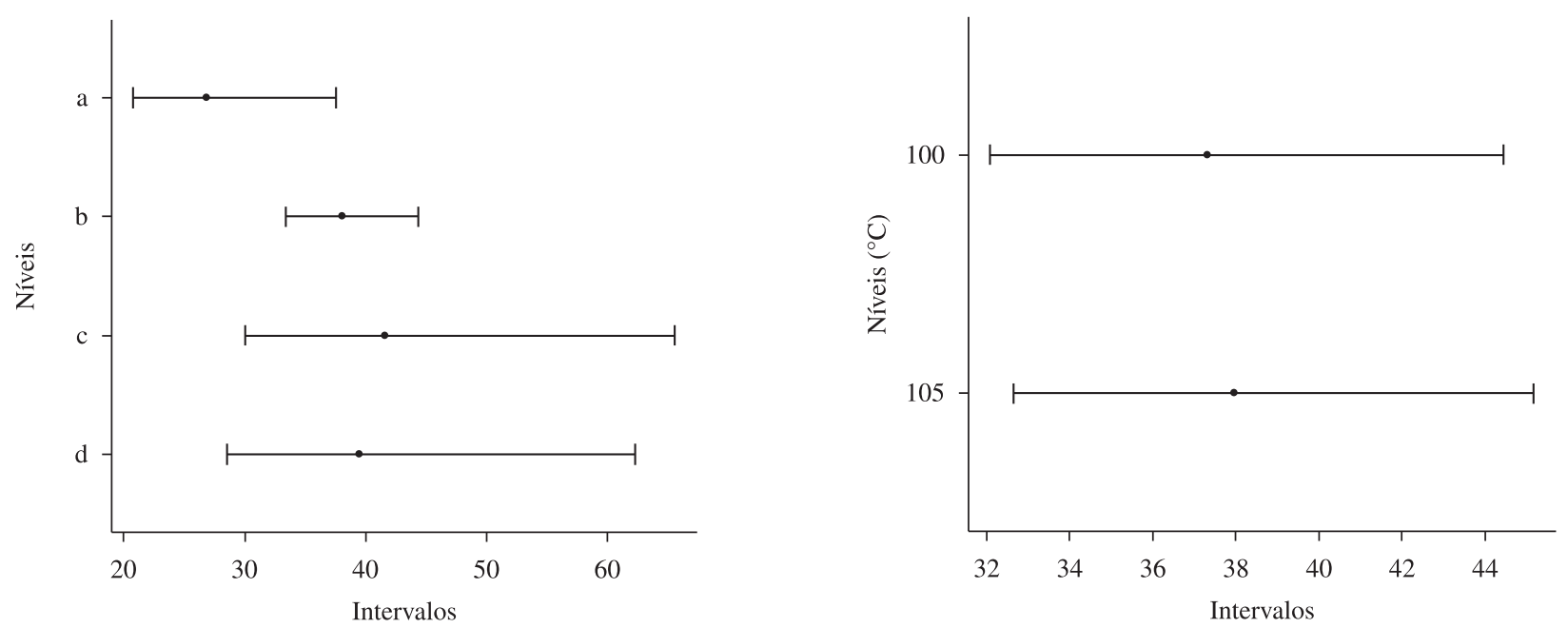

Figura 1. Intervalo de confiança para o desvio-padrão, fator temperatura $\left(\mathrm{a}=\mathrm{BOPP}\right.$ metalizado $1 / 100{ }^{\circ} \mathrm{C} ; \mathrm{b}=\mathrm{BOPP}$ metalizado $1 / 105{ }^{\circ} \mathrm{C} ; \mathrm{c}=\mathrm{BOPP}$ metalizado $3 / 100{ }^{\circ} \mathrm{C}$; e $\mathrm{d}=$ BOPP metalizado $3 / 105^{\circ} \mathrm{C}$ ).

Nesta etapa foram variados os fatores considerados mais impactantes nos resultados: amostra, analista e flutuação da temperatura no laboratório (que afeta a temperatura das barras da termosseladora). Com as 17 amostras apresentadas na Tabela 1 foram realizados 41 experimentos, cada um com cinco repetições. Estes experimentos foram executados por quatro analistas em temperatura ambiente variando entre 20 e $24^{\circ} \mathrm{C}$. Cada amostra foi avaliada pelo menos duas vezes.

Como nos experimentos foram utilizadas diferentes amostras, foi aplicada a técnica de CEP para pequenos lotes. A padronização dos resultados foi feita com os pares (mesma amostra), de modo a ter $99,73 \%$ dos valores entre \pm 3 desvios-padrão da média e da amplitude padronizadas.

\section{Resultados e Discussão}

A análise estatística dos resultados foi feita com o EstatcampExcel_Plus versão 1.6 e, em nenhum momento houve interesse em avaliar se os valores obtidos indicavam uma boa adesão do metal ao filme plástico.

\section{Método de ensaio - planejamento do experimento}

As variáveis que mais influenciaram na variabilidade dos resultados, ao nível de erro de 5\%, foram o tempo de termosselagem, o filme selante, o uso de fita e o ângulo de ensaio. Apesar da temperatura de termosselagem ter apresentado influência significativa, esta diferença está centrada em cada um dos blocos (amostras) e, ao avaliar-se a temperatura independentemente do bloco, não se detectou influência significativa $(\mathrm{P}$-valor $=0,849)$. $\mathrm{Na}$ Figura 1 são apresentados os gráficos representativos dos intervalos de confiança para o desvio padrão do fator temperatura de termosselagem com e sem a dependência do fator bloco, os quais ilustram esta dependência do bloco.

A variabilidade resultante da interação entre os fatores com maior influência na determinação da força de adesão foi analisada duas a duas, de forma a facilitar a interpretação dos resultados, os quais são apresentados na Tabela 3. A partir das combinações que resultaram em menor variabilidade, tem-se que a configuração com menor variabilidade ao nível de 5\% de significância foi: $90^{\circ}$ / EAA / sem fita / 5 segundos, podendo ser utilizadas as temperaturas de 
Tabela 3. Intervalo de confiança para o desvio padrão ao nível de 5\%.

\begin{tabular}{|c|c|c|c|}
\hline \multicolumn{4}{|c|}{ Intervalos de confiança para o desvio-padrão } \\
\hline Fator & Limite inferior & Desvio-padrão & Limite superior \\
\hline \multicolumn{4}{|c|}{ Selante /Fita (p-valor: 6,194 × 10-12) } \\
\hline EAA / sem fita & 13,73 & 16,92 & 21,83 \\
\hline PET/EAA / com fita & 22,81 & 28,10 & 36,26 \\
\hline PET/EAA / sem fita & 32,03 & 39,46 & 50,92 \\
\hline EAA / com fita & 34,84 & 43,00 & 55,60 \\
\hline \multicolumn{4}{|c|}{ Fita / Tempo (p-valor: 3,365 × 10-12) } \\
\hline Sem fita / 5 segundos & 13,73 & 16,92 & 21,83 \\
\hline Com fita / 5 segundos & 22,03 & 27,19 & 35,16 \\
\hline Sem fita / 15 segundos & 32,31 & 39,81 & 51,37 \\
\hline Com fita / 15 segundos & 34,64 & 42,68 & 55,08 \\
\hline \multicolumn{4}{|c|}{ Ângulo / Selante (p-valor: 3,764 × 10-2) } \\
\hline $90^{\circ} / \mathrm{EAA}$ & 37,63 & 46,37 & 59,83 \\
\hline $90^{\circ} / \mathrm{PET} / \mathrm{EAA}$ & 24,36 & 30,01 & 38,72 \\
\hline $180^{\circ} / \mathrm{EAA}$ & 27,88 & 34,35 & 44,33 \\
\hline $180^{\circ} / \mathrm{PET} / \mathrm{EAA}$ & 28,00 & 34,55 & 44,68 \\
\hline \multicolumn{4}{|c|}{ Ângulo / Fita (p-valor: 3,417 × 10 } \\
\hline $90^{\circ} / \mathrm{sem}$ fita & 21,91 & 27,00 & 34,83 \\
\hline $90^{\circ} /$ com fita & 36,29 & 44,71 & 57,69 \\
\hline $180^{\circ} / \mathrm{sem}$ fita & 31,07 & 38,28 & 49,40 \\
\hline $180^{\circ} /$ com fita & 25,06 & 30,92 & 39,99 \\
\hline \multicolumn{4}{|c|}{ Ângulo $/$ Tempo $\left(p\right.$-valor $\left.=3,767 \times 10^{-9}\right)$} \\
\hline $90^{\circ} / 5$ segundos & 18,31 & 22,56 & 29,11 \\
\hline $90^{\circ} / 15$ segundos & 39,02 & 48,07 & 62,03 \\
\hline $180^{\circ} / 5$ segundos & 21,72 & 26,80 & 34,66 \\
\hline $180^{\circ} / 15$ segundos & 25,57 & 31,51 & 40,66 \\
\hline \multicolumn{4}{|c|}{ Tempo $/$ Selante $\left(p\right.$-valor $\left.=3,365 \times 10^{-12}\right)$} \\
\hline 5 segundos / EAA & 13,73 & 16,92 & 21,83 \\
\hline 5 segundos / PET/EAA & 22,03 & 27,19 & 35,16 \\
\hline 15 segundos / PET/EAA & 32,31 & 39,81 & 51,37 \\
\hline 15 segundos / EAA & 34,64 & 42,68 & 55,08 \\
\hline
\end{tabular}

100 ou $105^{\circ} \mathrm{C}$. Frente a estes resultados e considerando que não houve fatores de ordem técnica que impedissem a adoção dos parâmetros de ensaio definidos pela análise estatística e que, dentre as duas temperaturas de termosselagem, a menor delas é a mais adequada para o filme de EAA, pois facilita a operação, reduz o retrabalho e o gasto com insumos, os parâmetros do método para determinação da força de adesão da metalização a filmes de BOPP recomendados foram:

- Ângulo de ensaio: $90^{\circ}$.

- Fita: sem fita para ancoragem do filme selante.

- Filme selante: EAA $25 \mu \mathrm{m}$.

- Tempo de termosselagem: 5 segundos.

- Temperatura de termosselagem: $100{ }^{\circ} \mathrm{C}$.

\section{Método de ensaio - avaliação da estabilidade/robustez}

Os gráficos de CEP referentes aos 41 experimentos realizados com as 17 amostras (Figura 2) demonstraram que o método de ensaio estabelecido é estável ao longo do tempo, quando comparado dentro das amostras, com todos os pontos se mantendo dentro dos limites estabelecidos. Neste caso, as propriedades estatísticas foram repetitivas ao longo do tempo.

Por meio de análise de variância constatou-se diferença significativa a $95 \%$ de confiança, na variabilidade entre as amostras $\left(\mathrm{P}\right.$-valor $\left.=1,446 \times 10^{-7}\right)$ e entre os substratos $\left(\mathrm{P}\right.$-valor $\left.=1,895 \times 10^{-21}\right)$.

Apesar deste trabalho não ter interesse em avaliar se os valores obtidos para a força de adesão do metal ao substrato são altos ou não, observou-se uma tendência de maior variabilidade nas amostras em que as maiores forças de adesão foram registradas, conforme apresentado no diagrama de dispersão (Figura 3). Como não foram encontradas evidências de que o analista e a temperatura ambiente no laboratório de termosselagem tiveram influência nesta variabilidade, ela pode ter como causa a própria amostra ou uma interação amostra/método decorrente de um ou mais parâmetros do método de ensaio.

Na Tabela 4 são apresentados os intervalos de confiança para o desvio-padrão da força de adesão do metal nas 17 amostras e nos substratos. Entende-se como substrato as diferentes superfícies (polímero/tratamento superficial) sobre as quais foi 


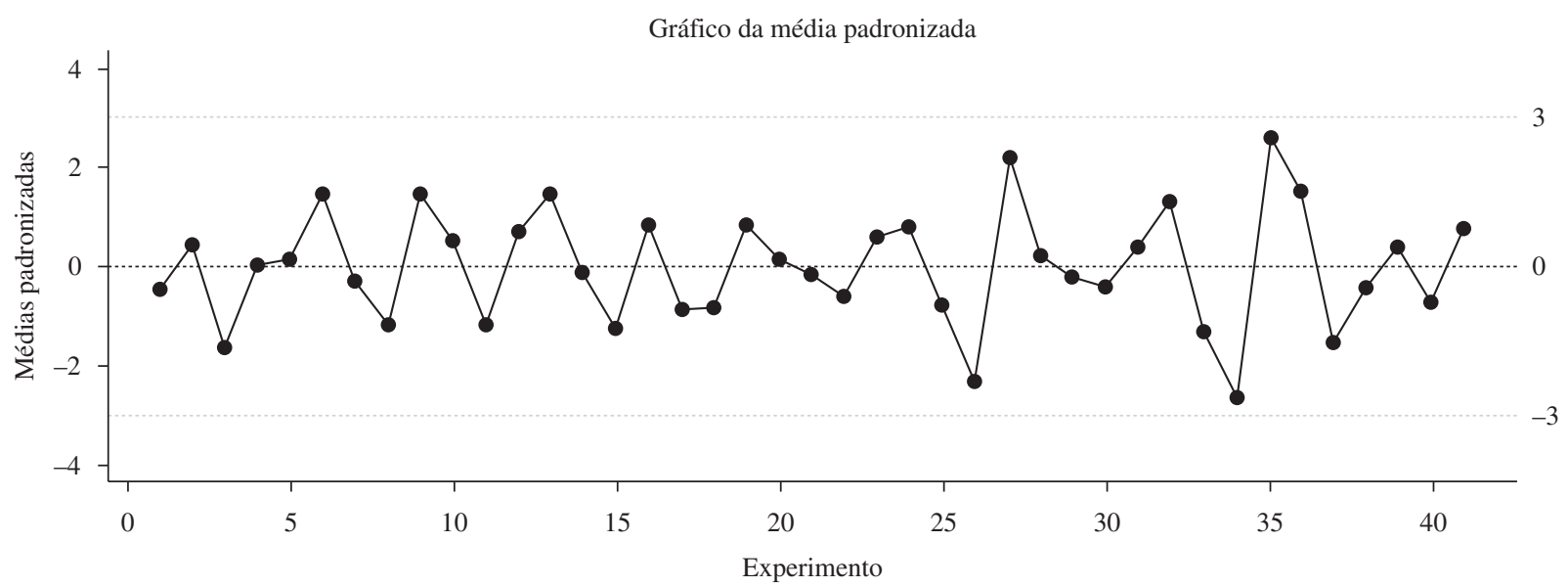

(a)

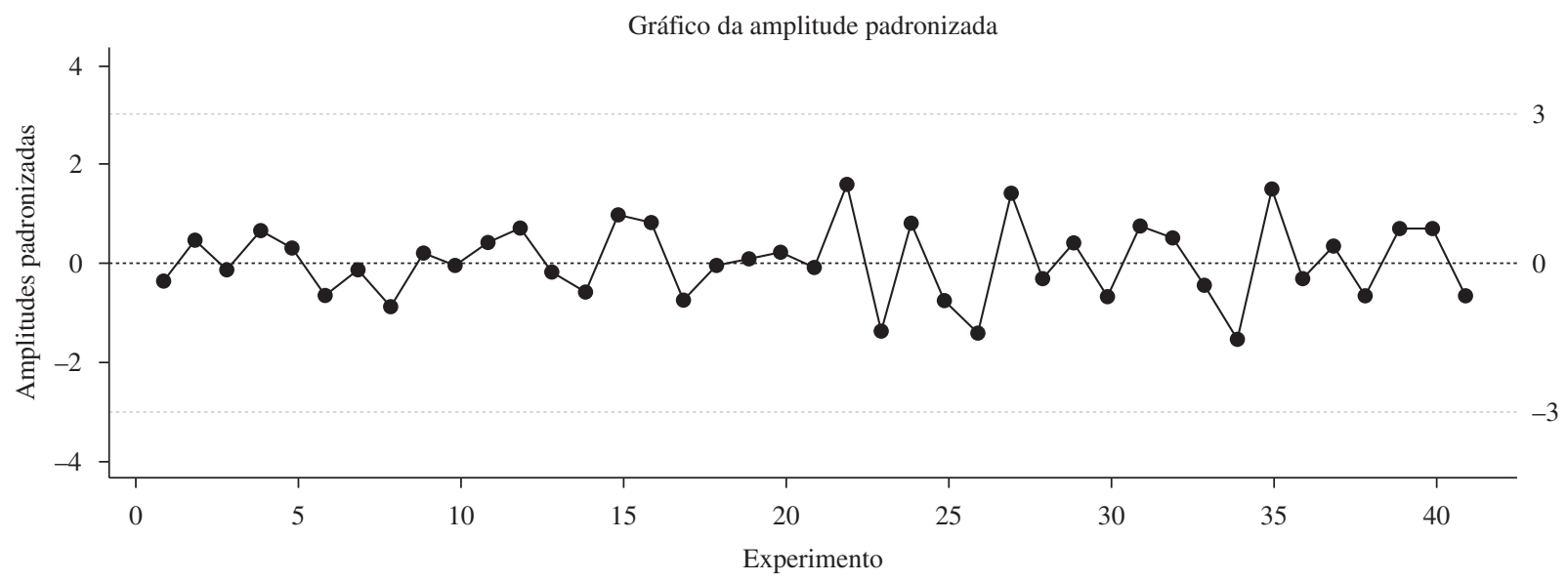

(b)

Figura 2. Gráfico de CEP para a) média padronizada; e b) amplitude padronizada.

aplicada a metalização, ou seja: BOPP metalizado - fabricante 1 , BOPP metalizado 5 - BOPP tratado por chama, fabricante 2, BOPP metalizado 6 - fabricante 2, PET coat - PET revestido por coating, PET coex - PET revestido por coextrusão, PET st - PET sem tratamento superficial, PET metalizado 10 - PET com superfície tratada para adesão intermediária e PET metalizado 11 - PET com superfície tratada para alta adesão.

Considerando de importância estatística valores de desviopadrão superiores a $25 \mathrm{~N} / \mathrm{m}$ (limite superior do intervalo de confiança para o desvio-padrão, previsto com $95 \%$ de confiança, na configuração proposta) e analisando os valores individuais de força de adesão obtidos e as demais informações disponíveis sobre as amostras/substratos, tem-se que as variabilidades críticas foram apresentadas pelas amostras: BOPP metalizado 4 - superfície tratada por corona e plasma, BOPP metalizado 5 - superfície tratada por chama, PET coat metalizado 2 - superfície revestida por coating e PET coex metalizado 9 - superfície revestida por coextrusão.

A amostra BOPP metalizado 4 foi a única, dentre quatro de um mesmo substrato (superfície tratada por corona mais plasma), na qual foi registrada variabilidade. O mesmo se aplica ao PET coat metalizado 2 e ao PET coex metalizado 9, em um universo de 3 amostras de cada substrato. Quando estas amostras são excluídas da análise de variância, apesar de ainda haver diferença significativa a $95 \%$ de confiança entre os substratos $\left(\mathrm{P}\right.$-valor $\left.=4,036 \times 10^{-13}\right)$,

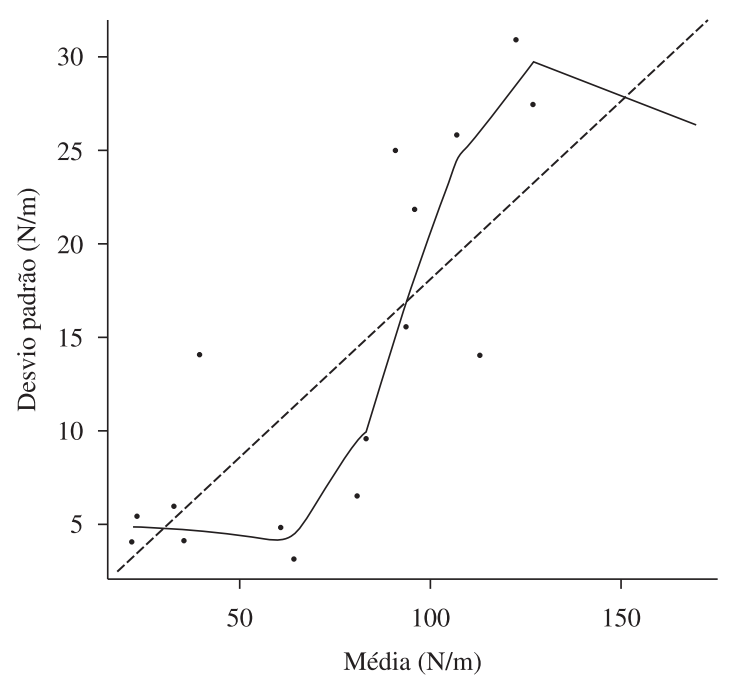

Figura 3. Diagrama de dispersão.

o desvio padrão para BOPP tratado por corona mais plasma, PET coat e PET coex metalizados fica abaixo de $25 \mathrm{~N} / \mathrm{m}$ (Tabela 5). Este resultado reforça a hipótese de que a causa da variabilidade esteja na amostra e não no tipo de substrato, o que não exige variações no método de ensaio em função do tipo de amostra. 
Tabela 4. Intervalo de confiança para o desvio padrão da força de adesão, ao nível de 5\% - amostra/substrato.

\begin{tabular}{|c|c|c|c|}
\hline \multicolumn{4}{|c|}{ Intervalo de confiança para o desvio-padrão - amostra } \\
\hline Amostra & Limite inferior & Desvio-padrão & Limite superior \\
\hline BOPP metalizado 2 & 1,945892275 & 3,150326262 & 7,175223713 \\
\hline PET st metalizado 4 & 3,703416252 & 5,320879099 & 9,019155521 \\
\hline *BOPP metalizado 5 & 19,17358014 & 25,75745155 & 38,29269731 \\
\hline *BOPP metalizado 4 & 18,36218122 & 27,46417887 & 50,90777918 \\
\hline PET coex metalizado 7 & 4,017311878 & 6,503876536 & 14,81331306 \\
\hline PET st metalizado 6 & 2,5028095 & 4,051954261 & 9,22878327 \\
\hline BOPP metalizado 6 & 9,619424222 & 15,5734853 & 35,47037092 \\
\hline BOPP metalizado 1 & 2,993293457 & 4,846029302 & 11,03737882 \\
\hline BOPP metalizado 3 & 5,90776807 & 9,564453867 & 21,78412345 \\
\hline PET metalizado 10 & 8,686568539 & 14,06322711 & 32,03058739 \\
\hline PET coat metalizado 1 & 15,40264284 & 24,93629831 & 56,79523455 \\
\hline *PET coat metalizado 2 & 19,08211812 & 30,89323011 & 70,36281923 \\
\hline PET coat metalizado 3 & 13,49351224 & 21,84548781 & 49,75556471 \\
\hline PET st metalizado 6 & 3,35295703 & 5,428311074 & 12,36359129 \\
\hline PET coex metalizado 8 & 8,65821672 & 14,01732658 & 31,92604375 \\
\hline *PET coex metalizado 9 & 16,28227236 & 26,36038535 & 60,03875355 \\
\hline PET metalizado 11 & 2,566489928 & 4,155050474 & 9,463596535 \\
\hline \multicolumn{4}{|c|}{ Intervalo de confiança para o desvio-padrão - amostra } \\
\hline Substrato & Limite inferior & Desvio-padrão & Limite superior \\
\hline BOPP metalizado 6 & 9,619424222 & 15,5734853 & 35,47037092 \\
\hline aBOPP metalizado $5^{*}$ & 19,17358014 & 25,75745155 & 38,29269731 \\
\hline 'BOPP metalizado* & 25,80965307 & 32,99117429 & 45,06280318 \\
\hline PET metalizado 10 & 8,686568539 & 14,06322711 & 32,03058739 \\
\hline PET coat metalizado* & 21,48646654 & 28,86454261 & 42,91190031 \\
\hline PET coex metalizado* & 30,67073704 & 41,20253065 & 61,25435318 \\
\hline PET st metalizado & 5,823407857 & 7,545439678 & 10,53772173 \\
\hline PET metalizado 11 & 2,566489928 & 4,155050474 & 9,463596535 \\
\hline
\end{tabular}

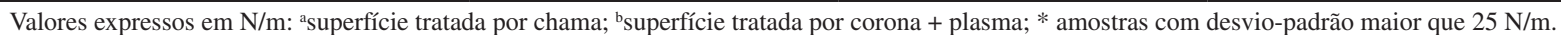

Tabela 5. Intervalo de confiança para o desvio padrão, ao nível de 5\%, para o substrato, expurgando as amostras BOPP metalizado 4, PET coat metalizado 2 e PET coex metalizado 9.

\begin{tabular}{|c|c|c|c|}
\hline \multicolumn{4}{|c|}{ Intervalo de confiança para o desvio-padrão } \\
\hline Substrato & Limite inferior & Desvio-padrão & Limite superior \\
\hline BOPP metalizado 6 & 9,619424222 & 15,5734853 & 35,47037092 \\
\hline${ }^{a}$ BOPP metalizado $5 *$ & 19,17358014 & 25,75745155 & 38,29269731 \\
\hline${ }^{\mathrm{b}} \mathrm{BOPP}$ metalizado & 8,774128295 & 11,78701019 & 17,5233335 \\
\hline PET metalizado 10 & 8,686568539 & 14,06322711 & 32,03058739 \\
\hline PET coat metalizado & 16,09605494 & 22,9369917 & 38,2172263 \\
\hline PET coex metalizado & 13,89840909 & 19,80533088 & 32,9993062 \\
\hline PET st metalizado & 5,823407857 & 7,545439678 & 10,53772173 \\
\hline PET metalizado 11 & 2,566489928 & 4,155050474 & 9,463596535 \\
\hline
\end{tabular}

Valores expressos em N/m: asuperfície tratada por chama; ${ }^{b}$ superfície tratada por corona + plasma; *amostra com desvio-padrão maior que 25 N/m.

\section{Conclusão}

O método desenvolvido para determinação da força de adesão da metalização com alumínio em filmes plásticos de BOPP e de PET foi considerado estável, robusto e replicável. A variabilidade apresentada por alguns substratos, ainda que menor que \pm 3 desvio-padrão, ou seja, confiança de $99,73 \%$, foi investigada e considerada inerente à amostra.
Duas das três etapas fundamentais para validação de um método de ensaio não normalizado foram executadas: o estudo de precisão (delineamento experimental e avaliação de desvio-padrão das medições) e a robustez. A terceira etapa consiste na avaliação da exatidão do método, o que requer um padrão de referência, ou um material de referência ou, pelo menos, a execução de medidas por meio de um método comprovadamente com maior qualidade analítica do que aquele que está sendo avaliado. Como nenhuma 
destas opções está disponível para a determinação da força de adesão da metalização, no atual cenário de conhecimento disponível, não poderá ser estudada a exatidão do método proposto.

\section{Agradecimentos}

Os autores agradecem à Dow Brasil, Inapel Embalagens Ltda., Polo Filmes, Terphane Ltda. e Vitopel do Brasil Ltda. pelo fornecimento dos insumos e à FAPESP e ao CNPq.

\section{Referências Bibliográficas}

1. Mueller, K. \& Weisser, H. - Pack. Tech. Sci., 15, p.29 (2002). http:// dx.doi.org/10.1002/pts.563

2. American Society for Testing and Materials - ASTM. - "ASTM F 904-98 - Standard Test Method for Comparison of Bond Strength or Ply Adhesion of Similar Laminates Made from Flexible Materials", ASTM, Philadelphia (2008).

3. Biron, M. - Enhancing polymer adhesion thanks to funcionalized materials and surface treatments. Polymer Additives \& colors (2008). Disponível em: http://www.specialchem4.polymers.com

4. Hernandez, R., Selk, S. E. M. \& Culter, J. H. D. - "Plastics Packaging - Properties, Processing, Applications and Regulations", Hanser, Munich (2000).

5. Loewy, M. - "Como se rompen los laminados?", Bogota (2005). Disponível em: http://www.envapack.com

6. Olafsson, G. \& Hildingsson, I. - J. Agric. Food Chem., 43, p.306 (1995). http://dx.doi.org/10.1021/jf00050a008

7. Bichler, C.; Mayer, K.; Langowski, H. C. \& Moosheimer, U. "Influence of Polymer Film Surfaces on Adhesion and Permeation Properties of Vacuum Web Coated High Barrier Films and Laminates: Results of a Cooperative Research Project", in: Proceedings of $41^{\text {st }}$ Annual Technical Conference, p.349, Philadelphia (1998).
8. Moosheimer, U.; Melzer, A. \& Langowski, H. C. - "European Thematic Network Vacuum Coating of Polymers Films for Packaging and Technical Applications", in: Proceedings of $44^{\text {th }}$ Annual Technical Conference, p.458, Philadelphia (2001).

9. Bichler, C.; Bischoff, H. C.; Langowski, H. C. \& Moosheimer, U. "The Substrate-process Interface in Barrier Film Coating", in: Proceedings of $39^{\text {th }}$ Annual Technical Conference, p.378, Tucson (1996).

10. Silvain, J. F.; Ehrhardt, J. J.; Picco, A. \& Lutgen, P. - "Crystallographic structure and adhesion of aluminum thin films deposited on mylar". in: Metallization of polymers, cap.33, Sacher E.; Pireaux J. J. \& Kowalczyk, S. P. (ed.), American Chemical Society, Washington (1990). http://dx.doi.org/10.1021/bk-1990-0440.ch033

11. Association of Industrial Metallizers, Coaters and Laminators "Metallizing technical reference - TP-105-92". 3. ed. South Carolina, AIMCAL.

12. European Metallizers Association. "Test procedure metal adhesion seal test". EMA (1999).

13. Associação Brasileira de Normas Técnicas - ABNT. "NBR ISO 15988 - Plásticos - Filme e revestimento - Filme de poli(tereftalato de etileno) (PET) biorientado". ABNT, Rio de Janeiro (2009).

14. Associação Brasileira de Normas Técnicas - ABNT. "NBR ISO 17555 - Plásticos - Filme e revestimento - Filme de polipropileno (PP) biorientado". ABNT, Rio de Janeiro (2009).

15. Leão, D. - "Planejamento de experimentos". Estatcamp, Campinas (2009).

16. Barros, C. B. - "Validação de métodos analíticos" Metrologia em química. Módulo 2. São Paulo (2008).

Enviado: 02/08/10

Aceito: 06/12/10

DOI: 10.1590/S0104-14282011005000038 